\title{
Pulsed Macroscopic Quantum Tunneling of Falling Bose Condensates
}

\author{
L. Salasnich ${ }^{1}$, A. Parola ${ }^{2}$ and L. Reatto ${ }^{1}$ \\ ${ }^{1}$ Istituto Nazionale per la Fisica della Materia, Unità di Milano Università, \\ Dipartimento di Fisica, Università di Milano, \\ Via Celoria 16, 20133 Milano, Italy \\ ${ }^{2}$ Istituto Nazionale per la Fisica della Materia, Unità di Como, \\ Dipartimento di Scienze Fisiche, Università dell'Insubria, \\ Via Lucini 3, 23100 Como, Italy
}

\begin{abstract}
We investigate macroscopic quantum tunneling of a Bose condensate and how it is affected by the interatomic interaction. We study the dynamics of a condensate falling under gravity and scattering on a Gaussian potential barrier that models a mirror formed by a far-detuned sheet of light. We observe bouncing, interference and quantum tunneling of the condensate. We find that the tunneling rate is very sensitive to the interatomic interaction and to the shape of the condensate. Under many conditions the tunneling rate is strongly enhanced by the interaction as achieved, for instance, by increasing the number of condensed particles. In a quasi 1D situation the tunneling pulse displays two peaks. The quantum tunneling can be quasi-periodic and in this way one could generate coherent Bose condensed atomic pulses. PACS numbers: 03.75.Fi, 32.80.Pj, 42.50.Vk
\end{abstract}

\section{INTRODUCTION}

Bose condensates, as achieved in trapped clouds of alkali-metal atoms [1-3], are ideal systems to study quantum phenomena at macroscopic level [4], like interference [5] and diffraction of matter waves. Tunneling is one of the striking quantum phenomena, which is important also in many applications. In the case of non-interacting particles at very low temperatures, when almost all particles are in the condensate, the tunneling of the condensate has the same character as in the case of a single particle. In this paper we address the question of how tunneling is affected by the interatomic interaction. The case of a condensate in a double-well potential has been already theoretically investigated and it is known that the interatomic interaction tends to suppress tunneling [6-10]. This effect can be so strong that under certain conditions it has been predicted that tunneling is suppressed completely and the condensate remains selftrapped in one of the minima of the potential. Here we show that under other conditions the tunneling rate can be enhanced by the interatomic interaction.

The case we study is that of a condensate impinging on a potential barrier that could model a mirror formed by a far-detuned sheet of light. We find that the interatomic interaction and the geometrical aspects of the system, like the aspect ratio of the cloud or the fact the the cloud remains trapped in the transverse directions, have a strong effect on the tunneling probability. This can be either enhanced or depressed and this effect can be very strong when the number of particles is large. The shape of the transmitted pulse can differ strongly from the initial shape and under certain conditions it gets a double peak structure.

The specific system we consider is a condensate falling under gravity and scattering on a Gaussian potential barrier. In a recent experiment, it has been shown the bouncing of condensates on the mirror formed by a fardetuned sheet of light when the strength of the energy barrier is much larger than the kinetic energy of the condensate [11]. Here we reproduce the bouncing effect and also predict that, when the energy barrier becomes comparable to the kinetic energy, macroscopic quantum tunneling can be observed. We consider the case in which the trapping potential in the horizontal directions remains active during the fall as well as the case in which the trapping potential is turned off at an initial time in all directions. We make comparison also with the behavior of a one dimensional case.

\section{NUMERICAL PROCEDURE}

The zero-temperature dynamics of a low-density Bose condensate can be accurately described by the timedependent Gross-Pitaevskii equation (TDGPE) [12,13] for the macroscopic wavefunction (order parameter) $\psi(\mathbf{r}, t)$ of the condensate in an external potential $V_{e x t}(\mathbf{r})$. When the wavefunction is normalized to unity then the parameter of the nonlinear term of the TDGPE is $\gamma=$ $4 \pi \hbar^{2} a_{s}(N-1) / m$, where $a_{s}$ the s-wave scattering length and $N$ is the number of condensed atoms.

In our problem, a Bose condensate is initially trapped in a harmonic potential and is under the action of gravity. In addition there is a Gaussian barrier centered at $z=0$. Therefore at $t \leq 0$ the external potential reads

$$
V_{e x t}^{<}(\rho, z)=\frac{m}{2}\left(\omega_{\rho}^{2} \rho^{2}+\omega_{z}^{2}\left(z-z_{0}\right)^{2}\right)+m g z+U e^{-\frac{z^{2}}{\sigma^{2}}},
$$

where $\rho=\left(x^{2}+y^{2}\right)^{1 / 2}$ and $z$ are the cylindrical coordinates, $\omega_{\rho}$ and $\omega_{z}$ are the frequencies of the trapping harmonic potential, $z_{0}$ is the position of the trap minimum along the $z$ axis, $g$ is the gravity acceleration, $U$ 
is the height of the potential barrier and $\sigma$ its width. First we consider the case in which an initially isotropic $\operatorname{trap}\left(\omega_{\rho}=\omega_{z}\right)$ is completely removed $\left(\omega_{\rho}=\omega_{z}=0\right)$ for $t>0$. Then, at positive time, $V_{\text {ext }}^{>}$only contains the gravitational term and the barrier. In cylindrical coordinates, the TDGPE equation becomes

$$
\begin{gathered}
i \hbar \frac{\partial}{\partial t} \psi(\rho, z, t)=\left[-\frac{\hbar^{2}}{2 m}\left(\frac{\partial^{2}}{\partial \rho^{2}}+\frac{1}{\rho} \frac{\partial}{\partial \rho}+\frac{\partial^{2}}{\partial z^{2}}\right)\right. \\
\left.+V_{e x t}^{>}(\rho, z)+\gamma|\psi(\rho, z, t)|^{2}\right] \psi(\rho, z, t),
\end{gathered}
$$

which is a nonlinear parabolic partial differential equation in $2+1$ dimensions and we study the time evolution starting from the ground state of the system with potential (1).

The numerical integration of this cylindrical TDGPE is performed using a modified split operator technique, adapted to the integration of a Schrödinger equation [14]. We write the Eq. (2) in the form

$$
i \hbar \frac{\partial}{\partial t} \psi(\rho, z, t)=\left[\hat{H}_{\rho}(\rho, z, t)+\hat{H}_{z}(\rho, z, t)\right] \psi(\rho, z, t),
$$

where

$$
\begin{aligned}
& \hat{H}_{\rho}(\rho, z, t)=\hat{T}_{\rho}+V_{e x t}^{>}(\rho, z=0)+\frac{2}{3} \gamma|\psi(\rho, z, t)|^{2}, \\
& \hat{H}_{z}(\rho, z, t)=\hat{T}_{z}+V_{e x t}^{>}(\rho=0, z)+\frac{1}{3} \gamma|\psi(\rho, z, t)|^{2},
\end{aligned}
$$

with $\hat{T}_{\rho}$ and $\hat{T}_{z}$ the transverse and axial kinetic operators, respectively. The full Hamiltonian is split in two sub-Hamiltonians, so that at each time we have to write the Laplacian and the external potential with respect to one coordinate only, leading to the solution of a tridiagonal system, and to huge savings in computer memory. Equation (3) is integrated using the scheme

$$
\begin{aligned}
& \psi(\rho, z, t+\delta)=\left[1+\hat{A}_{z}(t)\right]^{-1}\left[1-\hat{A}_{\rho}(t)\right] \\
& \times\left[1+\hat{A}_{\rho}(t)\right]^{-1}\left[1-\hat{A}_{z}(t)\right] \psi(\rho, z, t)
\end{aligned}
$$

where $\delta$ is the integration time step and $\hat{A}_{j}(t) \equiv$ $i \delta \hat{H}_{j}(\rho, z, t) /(2 \hbar)$ with $j=\rho, z$. The splitting is carried out so that the commutators are exact up to the order $\delta^{2}$ included. The nonlinear term $\gamma|\psi(\rho, z, t)|^{2}$ gives a problem, because we should really use a $\psi$ somehow averaged over the time step $\delta$, not a $\psi$ evaluated at the beginning of the time step. To circumvent this problem, we used a predictor corrector method. Each integration step $t \rightarrow t+\delta$ is performed twice: the first time we use $\psi(\rho, z, t)$ in the nonlinear term, obtaining a predicted $\tilde{\psi}(\rho, z, t+\delta)$; we then repeat the integration step, starting again from $\psi(\rho, z, t)$, but using $\frac{1}{2}(\psi(\rho, z, t)+\tilde{\psi}(\rho, z, t+\delta))$ in the nonlinear term. In this way the solution is accurate to $\mathrm{O}\left(\delta^{2}\right)$. At each time step the matrix elements entering the Hamiltonian are evaluated by means of finite-difference approximants using a typical mesh of $400 \times 2400$ points in the $(\rho, z)$ plane. As a check of the accuracy of the algorithm we find that in the time interval $\Delta t=10 \omega_{H}^{-1}$ the normalization of $\psi$ is conserved within $5 \%$ and the energy within few \%. Note that Eq. (3), now with the full potential (1), can be used with an imaginary time $t=i \tau$ to find the ground-state of the system.

In our calculations we adopt the harmonic oscillator units chosing $\omega_{H}=\left(\omega_{\rho}^{2} \omega_{z}\right)^{1 / 3}=2 \pi \times 100 \mathrm{~Hz}$. For ${ }^{23} \mathrm{Na}$ atoms, the harmonic length is $a_{H}=\left(\hbar /\left(m \omega_{H}\right)\right)^{1 / 2}=27$ $\mu \mathrm{m}$ and the scattering length is $a_{s}=3 \mathrm{~nm}$. The nonlinear term in scaled units is given by $4 \pi a_{s}(N-1) / a_{H}$.

\section{RESULTS AND DISCUSSION}

First we study the case in which the initial trap is isotropic $\left(\omega_{\rho}=\omega_{z}=\omega_{H}\right)$. In our computations we set $z_{0}=15 a_{H}$ so that the condensate is initially far from the Gaussian potential barrier whose effect is negligible. Actually, also the gravitational effect is negligible and the density profile of the ground-state has a Gaussian shape for a small number of atoms and it is an inverted parabola for a large number of particle. Then, we switch off the harmonic potential and use the previous wave-function as initial condition for Eq. (3). The total energy per particle of the condensate is about $180 \hbar \omega_{H}$ with $N$ ranging from 1 to $10^{5}$.

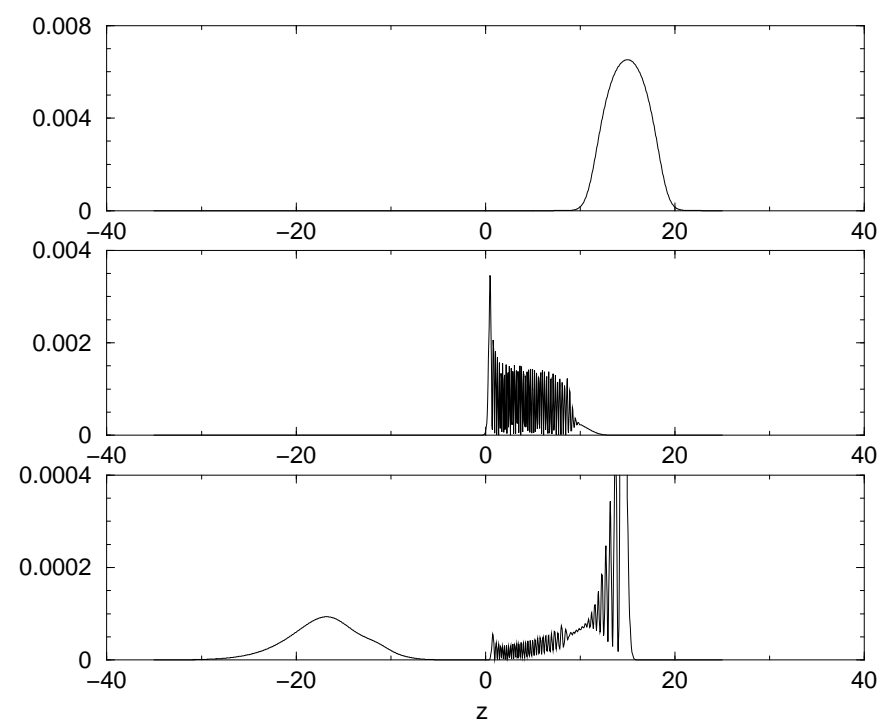

FIG. 1. Density profile $|\psi(\rho=0, z)|^{2}$ at $t=0, t=2$ and $t=4$. Barrier parameters: $U=200$ and $\sigma=1$. $N=10^{5}$ condensed atoms. Lenght in units $a_{H}=27 \mu \mathrm{m}$, time in units $\omega_{H}^{-1}=1.6 \mathrm{~ms}$ and energy in units $\hbar \omega_{H}$.

To simulate an impenetrable wall, we have chosen the 
following values for the parameters of the Gaussian potential barrier: $U=1000 \hbar \omega_{H}$ and $\sigma=a_{H}$. The center of mass motion of the condensate is quasi-periodic due to the bouncing of the condensate. Because the condensate is also expanding isotropically, the oscillation along the $z$ axis is damped and this damping increases with the number of atoms (larger repulsive interaction). We have verified that interference fringes appear when the condensate collides on the barrier.

The phenomenon of interference clearly appears also when the energy $U$ of the barrier is comparable to the chemical potential $\mu$ of the condensate defined as the total energy per particle plus the interaction energy per particle. The chemical potential, in contrast to the energy, is time-dependent [15]. Nevertheless, in our calculations $\mu$ is practically constant, apart a slight increase (no more than 4\%) during the collision time due to the enhanced density of the condensate. In Fig. 1, we plot the density profile of the condensate along the symmetry axis at three different instants. In this case the energy barrier is $U=200 \hbar \omega_{H}$ and the initial chemical potential $\mu=184.23 \hbar \omega_{H}$. Besides the interference between the incident wave-function and the reflected wave-function, one sees a transmitted wave, due to the quantum tunneling of the condensate.

For a non-interacting gas the semiclassical approximation of the linear Schrödinger equation predicts that the tunneling probability is proportional to $e^{-2 \int_{a}^{b} d z \sqrt{2 m\left(V_{e x t}^{>}(z)-\bar{E}\right) / \hbar^{2}}}$, where $a$ and $b$ are the classical turning points and $\bar{E}$ is the energy per particle of the condensate that, in the non-interacting case, coincides with the chemical potential $\mu$. In the interacting case, on the basis of a WKB study of the 1D GPE in the weak-coupling regime [8], it has been shown that the energy per particle $\bar{E}$ should be substituted by the chemical potential $\mu$ of the Bose condensate.

In general, the semiclassical quantization of the nonlinear Schrödinger equation appears a difficult task but numerical results can be easly obtained with our algorithm. As shown in Fig. 2, the effect of nonlinear term is such that the tunneling fraction $P_{T}$ increases with the number $N$ of condensed atoms, i.e. by increasing the chemical potential. For instance, for $N=10^{5}$ the tunneling probability is about 4 time larger than the noninteracting case. Note that, due to the high density in the impact region, the self-interaction becomes quite strong for a large number of particles and this clearly affects the tunneling fraction, as shown in Fig. 2.

While the transmitted wave-function of the condensate falls towards $z=-\infty$, the reflected wave-function goes back close to the initial position $z_{0}$ and then falls down again under the force of gravity. It means that, after a time roughly equal to the period $2 \sqrt{2 z_{0} / g}$, the condensate scatters again on the Gaussian barrier and now the tunneling fraction is smaller.

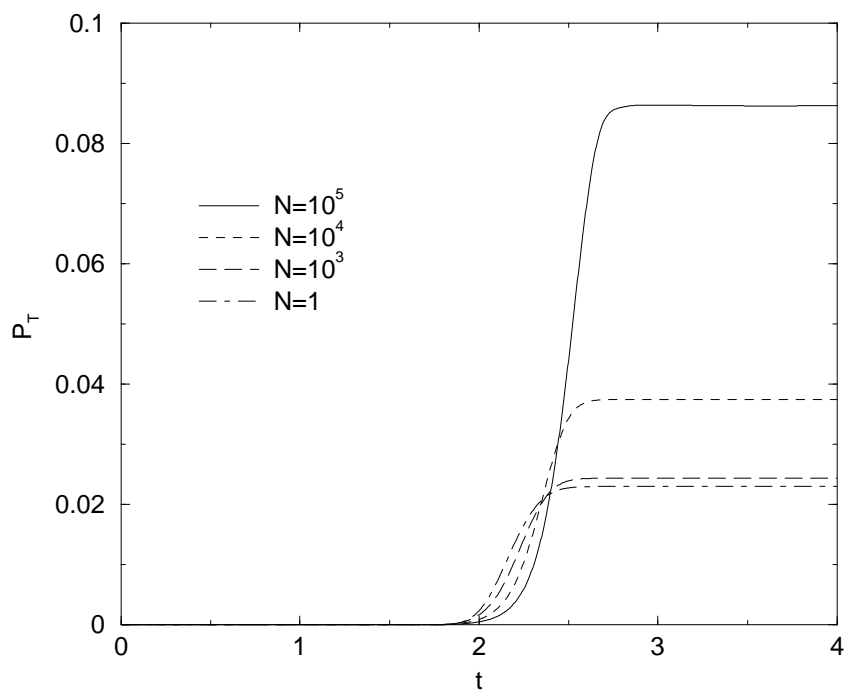

FIG. 2. Tunneling fraction $P_{T}$ as a function of time $t$. Barrier parameters: $U=200$ and $\sigma=1 . \quad N=1$ means non-interacting case. Initial position: $z_{0}=15$. Units as in Fig 1.

In Fig. 3 we plot the tunneling fraction $P_{T}$ as a function of time, showing that the macroscopic quantum tunneling of a Bose condensate falling under gravity is a quasi-periodic phenomenon. This shows that experimental set up similar to what we are considering should be able to generate coherent atomic pulses.

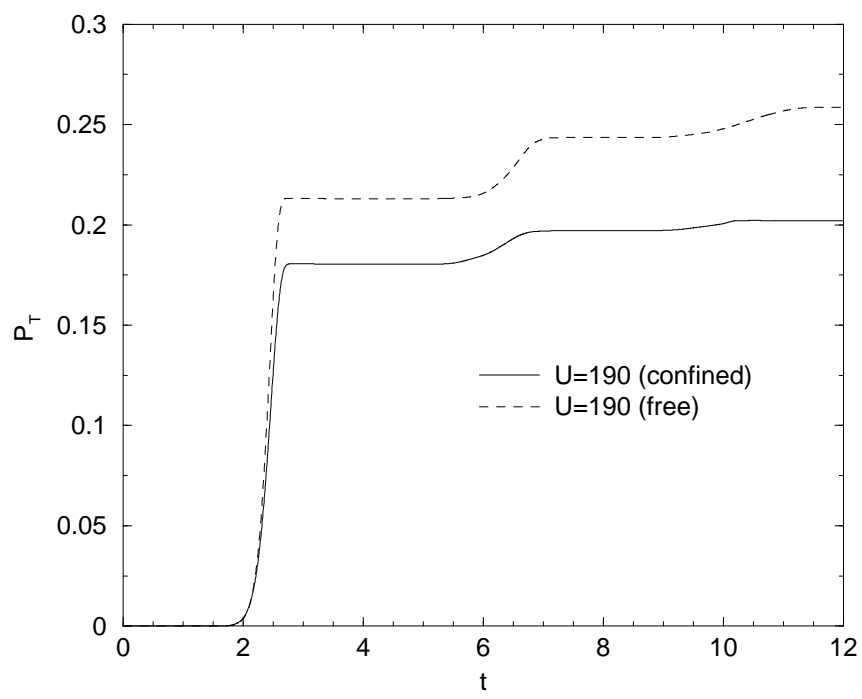

FIG. 3. Tunneling fraction $P_{T}$ as a function of time $t$. Comparison between radially-free $\left(\omega_{\rho}=0\right)$ and under radial confinament $\left(\omega_{\rho}=0.5\right)$ falling condensates. $N=10^{5}$ condensed atoms and initial position $z_{0}=15$. Barrier width: $\sigma=1$. Units as in Fig 1.

In the previous computation the condensate expands for $t>0$ in all directions but this leads to a decrease in density at the second bounce with a reduced tunneling fraction. It is interesting to see what happens if the condensate remains trapped in the transverse directions. We 
obtain this assuming that $V_{e x t}^{>}$does not confine $\left(\omega_{z}=0\right)$ in the $z$ direction but it remains confining $\left(\omega_{\rho}=\alpha \omega_{H}\right)$ in the horizontal directions.

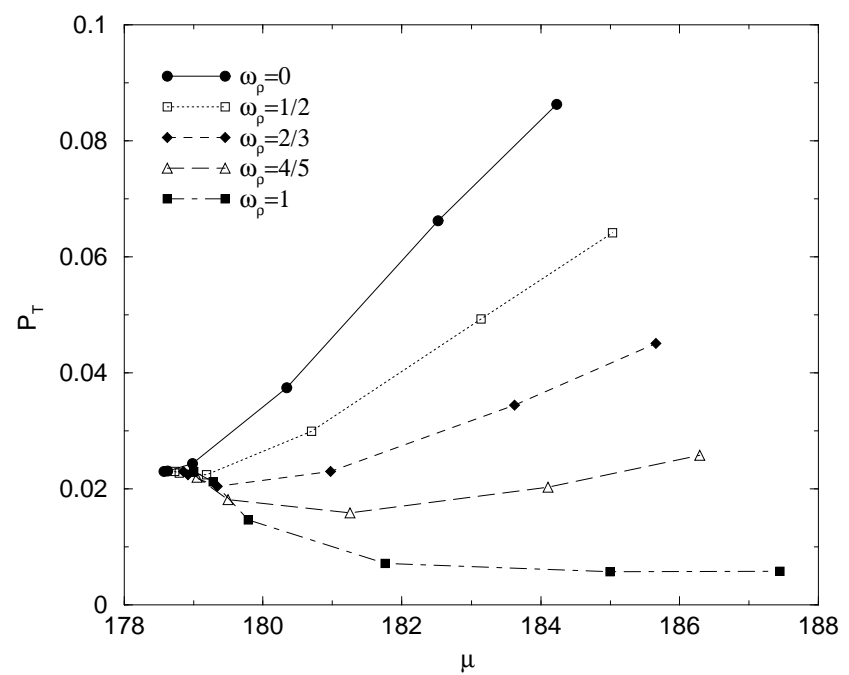

FIG. 4. Tunneling fraction $P_{T}$ as a function of the initial chemical potential $\mu$ for different frequencies $\omega_{\rho}$ of radial confinament. The points in each line correspond to a sequence of numbers $N$ of atoms. From left to right: $N=1,10^{2}, 10^{3}, 10^{4}, 5 \times 10^{4}, 10^{5}$. Barrier parameters: $U=200$ and $\sigma=1$. Units as in Fig 1 .

From Fig. 4 we see that, depending on the degree of this radial confinement, the interatomic interaction (and the chemical potential $\mu$ ) either enhances or depresses the tunneling probability $P_{T}$. Note that, in the present case, the system is not quasi-1D, due to the isotropic initial condition. In the case of strong radial confinament, the falling condensate shrinks in the radial direction during the motion. Such compression modifies the effect of the nonlinear term in the TDGPE. As shown in Fig. 4, the tunneling probability $P_{T}$ initially decreases and then increases with the chemical potential $\mu$.

In the previous calculations we have considered Bose condensates with isotropic initial conditions $\left(\omega_{z}=\omega_{\rho}\right)$. Often experimentally the confining potential of the trap is anisotropic. Here we investigate the case of cigarshaped initial conditions $\left(\omega_{z}=\omega_{\rho} / 10\right)$ which model a quasi-1D system. In this case we find that the tunneling fraction increases by increasing the number $N$ of particles both in the case of absence of confinement $\left(\omega_{\rho}=0\right)$ and in the case of strong radial confinement (Fig. 5). As shown in Fig. 5, due to the anisotropy of the trap, the axial elongation of the condensate grows with the number $N$ of particles and for $N=10^{4}$ one tip of the condensate touches the Gaussian barrier.

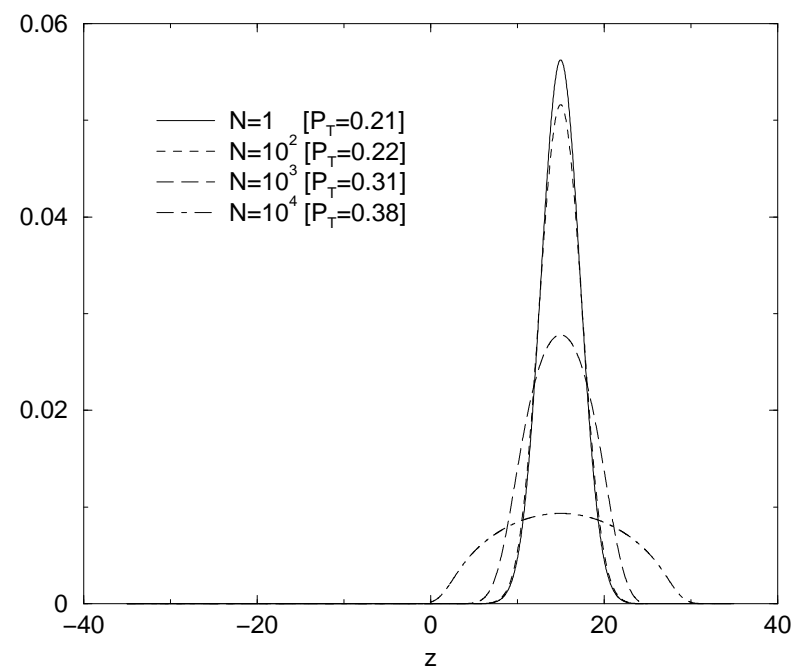

FIG. 5. Density profile $|\psi(\rho=0, z)|^{2}$ at $t=0$ for various values of the number $N$ of condensed atoms. Barrier parameters: $U=200$ and $\sigma=1$. Radially-confined $\left(\omega_{\rho}=1\right)$ falling condensate with cigar-shaped $\left(\omega_{\rho} / \omega_{z}=10\right)$ initial condition. $P_{T}$ is the tunneling fraction. Units as in Fig 1.

The exponential law suggested by Zapata et al. [8] for a 1D system seems able to capture the behavior of $P_{T}$ as a function of the chemical potential $\mu$ : on the basis of this law one finds a relative increment of the tunneling fraction of $87 \%$ as $N$ changes from 1 to $10^{4}$, whereas this relative increment is about $81 \%$ on the basis of the TDGPE.

In Fig. 6 we plot the density profile of the condensate at three time step. One sees that the transmitted pulse has a peculiar feature: the density profile clearly shows two peaks (see Fig. 6). The amplitude of the second slower peak increases with the number of particles.

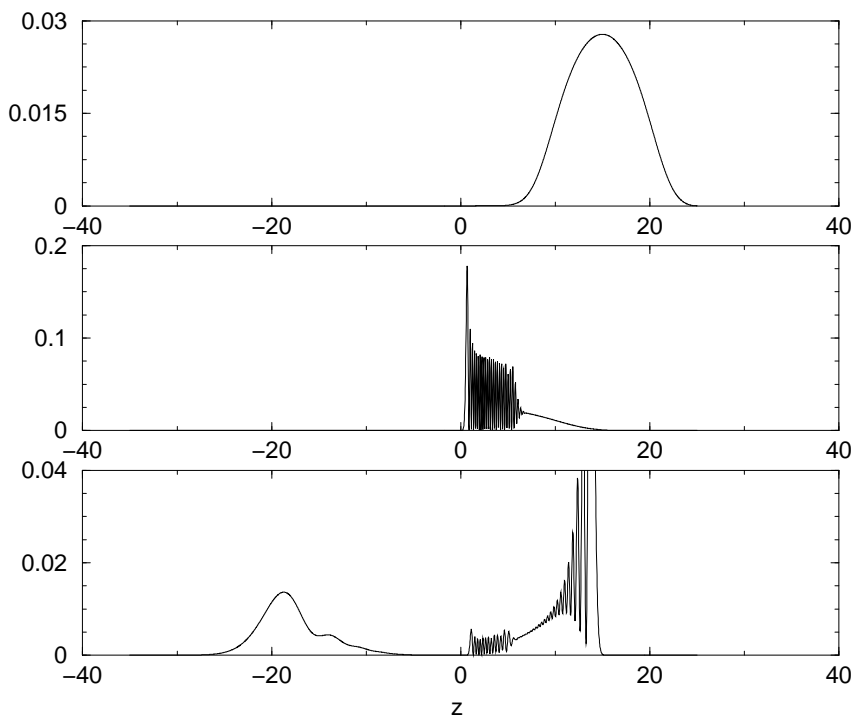


FIG. 6. Density profile $|\psi(\rho=0, z)|^{2}$ at $t=0, t=2$ and $t=4$. Barrier parameters: $U=200$ and $\sigma=1 . N=10^{3}$ condensed atoms. Radially-confined $\left(\omega_{\rho}=1\right)$ falling condensate with cigar-shaped $\left(\omega_{\rho} / \omega_{z}=10\right)$ initial condition. Units as in Fig 1.

Actually, for a large number of particles a third small peak can be detected. In the case of an isotropic cloud, the second peak shows up only with $N \geq 10^{5}$ particles (see Fig. 1). These two features of the tunneling of a cigar-shaped cloud, a tunneling rate enhanced by the interaction and the presence of a second peak in the transmitted pulse, are also present in the case of a purely one dimensional system.

For the sake of completeness, we have also considered the single-vortex state $\psi(\mathbf{r}, t)=\rho \phi(\rho, z) e^{i \theta}$ as initial condition but we have not found any significant difference in the dynamics of the condensate.

We have presented the results for the $\mathrm{Na}$ atom. As long as the scattering length is positive our results apply also to the other alkali-metal atoms by suitably rescaling the spatial and temporal scales and the number of particles.

\section{CONCLUSIONS}

We have studied the dynamics of Bose condensates falling under gravity and scattering on a Gaussian barrier that models a mirror of light. Apart the pure bouncing with interference, that can be seen for very large values of the energy barrier, we have investigated quantum tunneling. Our results show that macroscopic quantum tunneling of a Bose condensate should be observable experimentally and that the tunneling rate is very sensitive to the interatomic interaction and to geometrical features of the experiment. If initially the cloud is spherical under free fall, the tunneling fraction grows with the number of particles, which increases the interatomic interaction and the chemical potential of the condensate. But, the fraction is reduced by the interatomic interaction if the falling condensate is under a strong enough transverse confinement. In a $1 \mathrm{D}$ case, and in the case of a condensate with a cigar-shaped initial condition, the interatomic interaction always enhances the tunneling rate. Therefore the tunneling rate becomes larger and larger as the number of particles increases. In addition, in the 1D or quasi $1 \mathrm{D}$ case the density profile of the transmitted wave shows two peaks. We have also shown that in our system macroscopic quantum tunneling is a quasi-periodic phenomenon and it can be used to generate Bose condensed atomic pulses. One could generate truely periodic pulses if the bouncing part of the condensate is reconfined for a short period of time when it returns in the initial position and additional atoms are condensed in order to regenerate the initial state. Often the dynamics and static of a condensate is modelled by a $1 \mathrm{D}$ system. One important result of our computation is that geometrical aspects are important in tunneling phenomena and that $1 \mathrm{D}$ results not always reflect the behavior of 3D systems.

\section{REFERENCES}

[1] M.H. Anderson, J.R. Ensher, M.R. Matthews, C.E. Wieman, and E.A. Cornell, Science 269189 (1995).

[2] K.B. Davis, M.O. Mewes, M.R. Andrews, N.J. van Druten, S.D. Drufee, D.M. Kurn, and W. Ketterle, Phys. Rev. Lett. 753969 (1995).

[3] C.C. Bradley, C.A. Sackett, J.J. Tollett, and R.G. Hulet, Phys. Rev. Lett. 751687 (1995).

[4] F. Dalfovo, S. Giorgini, L. Pitaevskii, and S. Stringari, Rev. Mod. Phys. 71, 463 (1999).

[5] M.R. Andrews, C.G. Townsend, H.J. Miesner, D.S. Drufee, D.M. Kurn, and W. Ketterle, Science 275, 637 (1997).

[6] A. Smerzi, S. Fantoni, S. Giovannazzi and S.R. Shenoy, Phys. Rev. Lett. 79, 4950 (1997).

[7] G.J. Milburn, J. Corney, E. Wright and D.F. Walls, Phys. Rev. A 55, 4318 (1997).

[8] I. Zapata, F. Sols and A.J. Leggett, Phys. Rev. A 57, R28 (1998).

[9] S. Raghavan, A. Smerzi, S. Fantoni and S.R. Shenoy, Phys. Rev. A 59, 620 (1999).

[10] L. Salasnich, A. Parola, L. Reatto, Phys. Rev. A 60, 4171 (1999).

[11] K. Bongs, S. Burger, G. Birkl, K. Sengstock, W. Ertmer, K. Rzazewski, A. Sampera, and M. Lewenstein, Phys. Rev. Lett. 83, 3577 (1999).

[12] E.P. Gross, Nuovo Cimento 20(1961) 454; J. Math. Phys. 4, 195 (1963).

[13] L.P. Pitaevskii, Zh. Eksp. Teor. Fiz. 40, 646 (1961) [Sov. Phys. JETP 13, 451 (1961)].

[14] E. Cerboneschi, R. Mannella, E. Arimondo, and L. Salasnich, Phys. Lett. A 249, 245 (1998).

[15] C.W. Gardiner, Phys. Rev. A 56, 1414 (1997). 